Reuista

\title{
La formación docente y el uso de las tecnologías de información y comunicación como propuesta para cambiar las prácticas cotidianas tradicionales: una reflexión
}

Autor: Martha Diana Bosco Hernández

Teacher Training and the Use of Information and Communication Technology as a Proposal to Change Usual Practices in Education: A Reflectione

\section{Resumen}

La formación docente demanda la preparación y la actualización de los involucrados en los procesos de enseñanza, dadas las acciones y funciones que conlleva el trabajo cotidiano en los diferentes espacios aúlicos o virtuales. Para ello se requiere de una organización en el quehacer del profesor, que parta de la vinculación de todos los elementos y las acciones didácticas. Todo ello se tendrá que trabajar con base en la reflexión, la acción y el uso adecuado de las tecnologías de información y comunicación (TIC) con el propósito de generar la interacción y la interactividad entre los agentes educativos para construir el diálogo pedagógico. Con ello se propiciará el uso didáctico de las diferentes herramientas que nos brinda la tecnología.

Palabras clave: formación docente, TIC, tecnopedagogía, reflexión en la acción, educación abierta, educación a distancia

\section{Abstract}

Teacher training demands the necessary preparation and updating of those involved in the teaching process, given the actions and functions involved in daily work in different classrooms or online. That is why teachers have to be organized in what they are doing, and have to start by joining all the elements and didactic actions. All these elements have to be studied on the basis of the reflection about the action with the appropriate use of information and communication technology (ICT), in order to generate interaction and interactivity among educators, to build educational dialogue, thereby encouraging the use of available didactic technological tools.

Keywords: teaching training, ICT, technology and pedagogy, reflection in the action, traditional education, open education, distance education 


\section{Introducción}

De manera continua experimentamos transformaciones radicales en todos los ámbitos del quehacer humano. Muchas de ellas han sido posibles gracias al vertiginoso avance de las tecnologías de la información y de la comunicación (TIC), las cuales han producido una revolución en todos los medios sociales, entre los que se encuentra la educación en sus diferentes niveles y modalidades. Como sabemos, en la modalidad presencial se han incorporado algunas herramientas de estas tecnologías, mientras que en la modalidad abierta y a distancia el uso de medios ha alcanzado una destacada aplicación, ya que dichas herramientas, por sus peculiares características, favorecen los procesos de comunicación, el intercambio y el diálogo entre los agentes del proceso educativo. En la actualidad, las modalidades educativas diferentes de las presenciales se caracterizan por ser una propuesta innovadora en su metodología y también por ser promotoras del estudio independiente y autogestivo, el cual se apoya en materiales didácticos impresos y en las TIC. Por todo ello las prácticas de los docentes deben valorarse y actualizarse para enfrentar los retos que cada día nos demandan los avances del mundo actual.

Teniendo en cuenta que el objetivo principal de los docentes es preparar a los alumnos para la sociedad del conocimiento, proceso en el que las TIC juegan un papel determinante, se hace necesario considerarlas como un apoyo que propicie el desarrollo de habilidades que aprovechen, por una parte el uso adecuado de los medios y por otra, permitan la apropiación del modelo pedagógico que respalde su aplicación con propósitos educativos. Las peculiaridades de cada modelo educativo hacen indispensable que los docentes se formen para conocer y aplicar las estrategias didácticas adecuadas en el uso cabal de las TIC y de los entornos de aprendizaje, en tanto que las nuevas experiencias y competencias permitan propiciar que estas metodologías, medios y materiales innovadores se conviertan en una herramienta más efectiva que aquellas propias de la modalidad presencial.

$\mathrm{Al}$ ser la docencia parte nodal de los modelos pedagógicos, gracias a la aplicación de las TIC muchos profesores se enfrentan a la disyuntiva de continuar con sus prácticas y estilos docentes tradicionales o aprender e incorporarlas a su trabajo cotidiano. Las TIC incluyen todos aquellos dispositivos de los que nos podemos valer para interactuar con otros seres humanos a través de medios digitales y acceder, procesar, almacenar o difundir información en formato digital. ${ }^{1}$ Es sabido que la conectividad y la interactividad favorecen la planificación y el desarrollo de los procesos de enseñanza y aprendizaje debido a que propician la calidad y cantidad de entornos para el aprendizaje colaborativo, y por ende la multidireccionalidad, la libertad de edición y la difusión del trabajo. De ahí que las TIC, utilizadas como herramientas de comunicación, permitan al alumno realizar actividades de aprendizaje individual o colectivo mediante chats, foros, wikis, blogs, portafolios y redes sociales, entre otros, al mismo tiempo que asimila conocimiento. Lo anterior demanda que el diseño didáctico sea flexible y no lineal, que se inscriba en el mundo real y que en su propuesta se impulsen situaciones auténticas y significativas en las cuales la experiencia y el conocimiento sea compartido y adaptado colectivamente, con la aplicación de los princi-

\footnotetext{
1 Referidos a las tecnologías multimedia que contienen imágenes fijas y en movimiento, audio y textos e internet que facilita el acceso a los recursos y a los servicios educativos.
} 
pios derivados de los enfoques de la cognición situada, colaborativa y constructivista.

\section{El problema a atender}

A manera de ejemplo, se observa en diferentes reportes de investigación sobre el Sistema Universidad Abierta y Educación a Distancia (SUAyED) de los últimos 13 años (Fernández, 1999; Bosco y Molina, 2007; Cervantes, Herrera y Parra, 2011) que se alude al desarrollo heterogéneo de sus divisiones en ángulos: a) de la formación docente; b) de la práctica docente; c) del uso de las TIC; d) del desarrollo del material didáctico; e) de la tecnología, y f) de la administración escolar. En algunas divisiones del SUAyED estos aspectos que han resultado exitosos - en aquellas que han apostado a la innovación-, pero hay otras en las que aún existe poca claridad sobre su aplicación y en relación con los métodos de enseñanza apoyados en las tecnologías. El problema radica en que en el SUAyED y en otras instituciones educativas, los docentes que continúan con prácticas docentes basadas en una metodología tradicional tendrían que formarse en el uso didáctico de las TIC entendidas como: a) medio y recurso; b) mediadoras y facilitadoras del proceso de enseñanza-aprendizaje; c) apoyo metodológico, y d) apoyo a la tarea docente.

Es necesario revisar cómo ha evolucionado el aprovechamiento de dichas tecnologías como recurso didáctico en los aspectos antes mencionados tanto por los docentes de un sistema presencial como los de educación abierta y a distancia. En primer lugar, debe precisarse cuáles han sido las experiencias que se han distinguido por exitosas en el proceso y en segundo lugar, para que se propongan, si es el caso, nuevas formas de utilización de las TIC como herramientas de fortalecimiento en los procesos de enseñanza, aprendizaje y eva- luación. Las interrogantes básicas que surgen para fortalecerse en este sentido son:

- ¿Cómo aprovechan los docentes las Tic para propiciar ambientes de aprendizaje situados y significativos en entornos colaborativos flexibles?

- ¿Cómo crean los docentes ambientes interactivos de aprendizaje que promuevan el "aprender a aprender" a través del diálogo pedagógico y del trabajo colaborativo en función de las tecnologías?

- ¿Cómo se fomenta que los alumnos valoren críticamente sus contribuciones y las de sus compañeros para que todos los esfuerzos concurran en una autoevaluación formadora con apoyo de las Tic?

- ¿Cómo se propician con el empleo de las TIC actividades de aprendizaje basadas en las estrategias de la resolución de problemas, estudios de caso, investigaciones propositivas en la red, el uso del portafolios, entre otras?

- ¿Cómo se usan los recursos que ofrece la web, como bibliotecas, mapotecas, redes sociales, comunidades de diálogo, etcétera, con apoyo de las TIC?

- ¿Cuál es el uso de las Tic cuando se evalúa el proceso de aprendizaje en las modalidades presencial, abierto y a distancia?

Es preciso que los docentes conozcan los usos que hacen de las TIC en su práctica para poder reflexionar y valorar su aplicación y aprovechamiento en los entornos de aprendizaje presenciales, semipresenciales y a distancia como recursos didácticos que potencian y promueven el aprendizaje. De esa manera identificarán las mejores prácticas para socializarlas en la comunidad académica de su institución o bien para incorporarlas a su quehacer cotidiano en los espacios áulicos o virtuales. Con esto se pretende que haya nuevas formas de 
utilización de las TIC como herramientas de fortalecimiento en los procesos de enseñanza, aprendizaje y evaluación.

Desde esa óptica las TIC pueden ser utilizadas por aquellos docentes interesados en cambiar sus prácticas comunes, pues con el uso de estas herramientas podrán promover procesos de enseñanza-aprendizaje activos, constructivos y situados en la realidad. Por supuesto que es necesaria una formación continua centrada en el aprendizaje de y para el uso de las TIC como recurso didáctico. De esa manera los docentes deben considerar que las tecnologías puestas a su disposición no deben constituirse como un mero transmisor de información y conocimientos, y tampoco como un fin en sí mismo, sino que deben ser auténticos desafíos y alternativas de trabajo entre ellos y los alumnos para ayudarlos en la construcción de su propio conocimiento.

Cabe mencionar que los profesionales de la educación deben ocuparse con una diversidad de funciones entre las que han de considerarse las bases teóricas, la orientación metodológica y las competencias prácticas, además del uso de los medios. Por ello se hace necesario que la acción docente considere tres enfoques teórico-metodológicos en su quehacer: la concepción de la tecnopedagogía, la concepción del profesor reflexivo y la teoría de sistemas. Éstos son una forma adecuada de acercarse a los problemas que genera el modelo complejo de la educación presencial, abierta y a distancia desde el uso de las tecnologías en la práctica docente, con base en la reflexión sobre el quehacer docente en un entorno determinado, abierto y flexible, y la revisión de ese entorno como un sistema.

Esta mirada conjunta permite aproximar una visión constructivista desde el uso de las tecnologías de la enseñanza y el aprendizaje mediante la conectividad e interacción, sin excluir las ideas fundamentales del logro de los ob- jetivos, del control, de la evaluación del proceso y de las metas deseadas. Según Coll (2004), la concepción de la tecnopedagogía se explica en: a) los procesos formales escolares de la enseñanza y el aprendizaje en términos de "estructuras de participación" o sistemas de reglas para ayudas conjuntas y de su evolución a lo largo de los procesos de enseñanza-aprendizaje, y b) la potencialidad semiótica, relacionada con las representaciones, procesos, intervenciones e informaciones.

La tecnopedagogía se genera por las características de las TIC, ya que a través de ellas se crean diseños tecnológicos del proceso formativo y de manera conjunta con el diseño pedagógico o instruccional del proceso educativo, esto representa la síntesis de ambos y constituye parte de los referentes en los que se va a construir el proceso formativo. Coll (2004) afirma que los procesos de enseñanza-aprendizaje basados en las Tic originan relaciones que se configuran en un triángulo didáctico, entre el contenido que es objeto de enseñanza y aprendizaje, la actividad educativa e instrucciónal del profesor, y las actividades de aprendizaje de los alumnos. En ese triángulo se construirán las interacciones conjuntas entre los agentes educativos y se le otorgará significado a las acciones realizadas y a las ayudas sostenidas y ajustadas al proceso de construcción del conocimiento.

La concepción del profesor reflexivo, en la que Schön (1998) presenta el concepto de practicum reflexivo incluye tres procesos: a) conocimiento en la acción: capacidad natural del ser humano de saber hacer o resolver problemas mediante un conocimiento técnico, semiautomático y rutinario, fruto de la experiencia y de la reflexión personal anterior; $b$ ) reflexión en la acción: diálogo que la persona establece con la acción en curso y que demanda una solución, apoyándose para eso en esquemas, principios y convicciones de modo 
no riguroso ni sistemático, y c) reflexión sobre la acción y sobre la reflexión en la acción: que realiza la persona cuando, después de la acción a evocar, reconsidera y evalúa con distanciamiento crítico y emotivo, y con instrumentos adecuados de análisis.

La práctica docente requiere de un espacio de aplicación de los principios teóricos de la formación inicial, así como de un lugar de investigación, de reflexión y de elaboración del conocimiento pedagógico. Schön (1998) señala que el docente debe definir el problema a partir de su propia construcción y de la situación que enfrenta y asumir, de acuerdo con Coll (2004), que no basta con incorporar las tecnologías en las asesorías sin una estrategia que garantice el aprendizaje. Por otro lado, deberá considerarse el concepto de aprendizaje mediante la experiencia trabajada por John Dewey (2000), en la que el estudiante valora los conocimientos como necesarios y útiles para su vida cotidiana. Así, el aprendizaje mediante la resolución de problemas puede concebirse como un motor para la práctica y la reflexión, es decir, como un estímulo para pensar, un cordón para recurrir a conocimientos previos que ayuden a alcanzar el objetivo propuesto. Por su parte, Henderson (1992) plantea que un profesor reflexivo también es constructivista porque se pregunta cómo aprenden sus alumnos y propicia la participación activa y con ello el aprendizaje. Moreno (2011) señala las competencias prácticas del docente: a) ser diseñador de ambientes, procesos y recursos educativos; b) ser orientador de los procesos educativos; c) ser gestor de actividades administrativas cuando éstas sean de apoyo a sus labores académicas, y d) ser un investigador reflexivo de los procesos educativos, para poder fundamentar los proyectos docentes.

Para entender las TIC en la labor docente Galvis (2008) comenta que se emplean para: a) mejorar la productividad individual, simplificando actividades y ampliando nuestra capacidad personal; b) mediatizar la interacción con otros individuos o con grupos dialogando sincrónica o asincrónicamente en la red; c) explorar conjeturalmente objetos de estudio, apoyando la indagación, construcción y expresión de conocimiento; d) apoyar labores educativas, como la preparación de clases y pruebas, y la creación y administración de ambientes de aprendizaje, y e) ampliar el acervo cultural, científico y tecnológico, manteniendo actualizados a los usuarios en aquello que les interesa, a partir de la consulta de fuentes directas. Al llevar a la práctica esta perspectiva, se hace necesario que el docente vincule y articule en su trabajo cotidiano la asignatura que imparte con las características, antecedentes, necesidades e intereses de los alumnos, así como las reflexiones sobre sus propias necesidades, creencias y valores de su práctica en los diferentes espacios de enseñanza.

En la teoría de sistemas, el proceso educativo permite, por un lado, contemplar la actividad educativa como una acción ordenada, secuencial y sistémica, y por otro, como una estructura social compuesta por elementos humanos, institucionales y funcionales que confluyen en el país o territorio. En ese sentido, el sistema es el conjunto de partes o elementos vinculados entre sí por interacciones dinámicas, que se organizan en la consecución de objetivos comunes. Esta perspectiva aplicada a la educación se entiende como un conjunto de fenómenos interrelacionados entre los individuos y el mantenimiento del sistema social para intervenir en él de manera crítica, reflexiva y creativa, promoviendo y generando transformaciones que alimentan al propio sistema educativo. Para lograrlo será patente la conjunción de elementos para que se gesten en la práctica docente ambientes de aprendizaje que favo- 
rezcan los procesos cognitivos, los caminos a seguir y la elección de los temas que se proponen trabajar.

La teoría, la metodología y la práctica tendrá que cristalizar en un diseño didáctico basado en las TIC, que deberá inspirar la reflexión en la acción, para enriquecer las construcciones y procesos que se incuben durante el proceso de formación de los agentes educativos, y con ello la valoración permanente del quehacer del profesor para corregir, mejorar y actualizar la propia práctica. Dado que los docentes son los actores principales y responsables de generar los contenidos y de fomentar el desarrollo de competencias en los procesos de aprendizaje propuestos con base en los proyectos educativos basados en las TIC, tendrán que crear procesos de aprendizaje basados en los alumnos por medio de un modelo pedagógico en el cual la planeación didáctica se dirija al intercambio de conocimientos, de saberes y de metodologías para la recreación del conocimiento.

\section{La web 2.0 como recurso didáctico}

Se puede definir a la web 2.0 como recurso didáctico por el conjunto de herramientas que contiene y que permite pasar del trabajo individual al colectivo, ya que propicia la interacción entre el alumno, el contenido y el docente por medio de ambientes de aprendizaje que gestionan el conocimiento. Esta herramienta tiene la cualidad de posibilitar en el trabajo cotidiano de los alumnos habilidades como el aprendizaje autónomo y el trabajo colaborativo, crítico y creativo, así como acciones para los diferentes estilos de aprendizaje —que se reflejará en los medios de información y comunicación propuestos-, y la interactividad entre los agentes educativos y la participación en actividades grupales. La web 2.0 es multidireccional y permite la libertad de edición y de contenidos textuales y audiovisuales - hipertextualidad-. Pueden desarrollarse nuevas actividades de aprendizaje y de evaluación y generar la creación de redes de aprendizaje.

La web 2.0 cuenta con aplicaciones en línea que se crean gracias a los procesadores de texto, las hojas de cálculo, las bases de datos y los calendarios. También contiene herramientas de publicación, como blogs, webquest, mapas conceptuales y wikis. Para la gestión de la información cuenta con los indicadores de contenidos, páginas de inicio y marcadores sociales. Esto propicia entre los alumnos: a) procesos de creatividad e innovación; b) comunicación y colaboración entre los miembros de la comunidad; c) fomento de la investigación y el manejo de información; d) florecimiento del pensamiento crítico; e) caminos para la solución de problemas y toma de decisiones; $\mathrm{f}$ ) la práctica de una ética digital, y g) comprensión, apropiación y aplicación de las TIC en los diversos entornos educativos y sociales.

En la práctica docente en entornos virtuales las herramientas que más se utilizan son: el weblog, la wiki y las redes sociales. El weblog es una herramienta que permite la publicación personal y colectiva en la web. Al docente le concede la publicación de contenidos para la enseñanza a partir de la hipertuxtualidad en diversos formatos digitales, al mismo tiempo que invita a la reflexión personal y social de los alumnos sobre los contenidos de aprendizaje y promueve el "aprender a aprender" y la metacognición como una condición natural de desarrollo activo, constructivo y situado en la realidad en la conformación del conocimiento. Es una herramienta que tiene la ventaja de coadyuvar a la libre expresión, la creación y la colaboración en la construcción del conocimiento y a su publicación.

La wiki cuenta con páginas que pueden ser editadas por varias personas. Permite al docente propiciar el aprendizaje colaborativo a partir 
de la estimulación de la producción de textos colectivos encauzados a producir conocimientos. El alumno juega el papel de editor de su propio conocimiento y del de sus compañeros, logrando una producción de conocimiento colectivo, participa en el establecimiento de reglas de ayudas conjuntas y de su evolución durante los procesos de enseñanza-aprendizaje. De igual manera, potencializa la capacidad semiótica relacionada con las representaciones, procesos, intervenciones e informaciones entre docentes y alumnos.

Las redes sociales constituyen una estructura social compuesta por grupos de personas conectadas mediante una aplicación de internet por intereses comunes. Entre sus posibilidades destaca que facilita al docente el seguimiento personalizado del alumno en su proceso de aprendizaje. Se distingue porque el alumno se expresa por sí mismo, establece relaciones con otros y atiende a sus necesidades de formación. También motiva la colaboración grupal y se pueden compartir materiales y crear productos digitales. Facilita la inter e intra comunicación con grupos propios y ajenos que comparten los mismos intereses.

\section{Competencias que se desarrollan en los procesos educativos con el uso de la web}

Gracias al uso de las TIC en los programas educativos, se predispone el desarrollo de competencias digitales necesarias en la era de la información y la comunicación y en particular en la educación como la navegación y búsqueda de información en la web: la selección, la valoración, el procesamiento de la información, la expresión, la comunicación, el trabajo con otros y la gestión de ambientes virtuales de aprendizaje. Las competencias de orden social que se desarrollan son el trabajo en equipo, el respeto entre la comunidad, la tolerancia y la responsabilidad y, otras competencias como el aprendizaje autónomo, la capacidad crítica, la imaginación, la creatividad, la adaptación a un entorno cambiante, la resolución de problemas y la iniciativa.

El uso de las herramientas y el desarrollo de competencias propician la interactividad y la interacción dentro de una plataforma virtual educativa en la que se establece un diálogo didáctico continuo entre los agentes del proceso educativo. En las plataformas se podrán colocar contenidos académicos, imágenes y videos, entre otros elementos. Asimismo, se trabajará con metodologías de estudio específicas de cada área del conocimiento, para lo cual se construirá un ambiente de aprendizaje propicio para las diversas comunidades que se gesten. Se encontrarán las herramientas que hemos mencionado y otras para que los alumnos se incorporen a su curso de una manera atractiva, motivante e interactiva. En ese sentido se cuidará el diseño del trabajo a construir en la misma planeación didáctica.

En las plataformas se cuenta con un sistema de administración del curso, que puede ser dado de alta o de baja. También hay un espacio para el registro de los alumnos, asesores y tutores. Pueden colocarse diversas ligas a páginas web con multimedia, glosarios automáticos, bibliotecas virtuales, repositorios. Para la interacción de los docentes y alumnos se crean foros, chats, grupos de trabajo, actividades de aprendizaje individuales y colaborativas, páginas específicas para los alumnos, autoevaluaciones, exámenes con registro, bandeja de entrega de tareas de alumnos, seguimiento de alumnos, registro de acceso y sus estadísticas, todo ello administrado y regulado por el docente. Finalmente con toda esta consideración se pretende que los docentes reflexionen sobre la importancia de formarse y actualizarse para cambiar el traba- 
jo cotidiano y tradicional, a una propuesta educativa más amplia con el uso de las diferentes herramientas que nos ofrece la web, con ello se enriquecerá el trabajo al crear espacios de reflexión y evaluación continua de manera colegiada, pues se fortalecerá e innovará la práctica docente de cara a los retos que s se presentan día a día.

\section{Referencias}

Bosco, M. D. y Molina, M. (2007). Diagnóstico del Sistema Universidad Abierta y Educación a Distancia de la unam. México: Coordinación de Universidad Abierta y Educación a DistanciaUniversidad Nacional Autónoma de México.

Cervantes, F., Herrera, A. y Parra, P. (2011). Situación Actual del Sistema Universidad Abierta y Educación a Distancia. En Cervantes, F., Herrera, A. y Parra, P. Prospectiva del Sistema Universidad Abierta y Educación a Distancia de la unam: Tres rutas para su consolidación al año 2021 (pp. 39-49). México: Coordinación de Universidad Abierta y Educación a DistanciaUniversidad Nacional Autónoma de México.

Coll, C., Mauri, T. y Onrubia, J. (2010). La utilización de las Tic en la educación: del diseño tecnopedagógico a las prácticas de uso. En Coll, C. y Monereo, C. (eds.). Psicología de la educación virtual. Enseñar y aprender con las tecnologías de la información y la comunicación (pp. 74-104). Madrid: Morata.

Coll, C. (2004). Psicología de la educación y prácticas educativas mediadas por las tecnologías de la información y la comunicación: una mirada constructivista. En Sinéctica, 25, 1-24.
Coordinación Universidad Abierta y Educación a Distancia (CUAED). (2012). Sistema de Universidad Abierta y Educación a Distancia. En línea: http://distancia.cuaed.unam.mx/40aniversario/. Consultado el 10 de octubre de 2012.

Dewey, J. (2000). Experiencia y educación. Buenos Aires: Lozada.

Fernández, J. et al. (1999). Situación actual. Programa de Transformación del sua. Cuaderno de trabajo. México: Sistema de Universidad Abierta-Universidad Nacional Autónoma de México.

Galvis, A. H. (2008). La piola y el desarrollo profesional docente con apoyo de tecnologías de información y comunicación. Bogotá: Metacursos.

Henderson, J. (1992). Reflective Teaching: Becoming and Inquiring Educator. Nueva York: Macmillan.

Moreno, M. (2011). Por una docencia significativa en entornos complejos. México: Universidad de Guadalajara.

Novo, M. et al. (2002). El enfoque sistémico: su dimensión educativa. Madrid: Universidad Nacional de Educación a Distancia.

Schön, D. (1998). El profesional reflexivo. Cómo piensan los profesionales cuando actúan. Barcelona: Paidós.

\section{Autor}

\section{Martha Diana Bosco Hernández}

Presidenta de la Academia Mexicana de Profesionales de Educación Abierta y a Distancia, Vicepresidenta de Innovación de la Academia Mexicana de la Ciencia en Sistemas, Académica de la Facultad de Filosofía y Letras de la Universidad Nacional Autónoma de México, bosco@servidor.unam.mx 